\title{
Aproximación a los estudios de la frontera hispano-portuguesa
}

\section{Approach to the Studies of the Spanish-Portuguese Border}

\author{
María Victoria Navas Sánchez-Élez [mvnavas@ucm.es] \\ Universidad Complutense de Madrid, España \\ Centro de Linguística da Universidade de Lisboa, Portugal
}

\begin{abstract}
RESUMEN
Mediante este trabajo presentamos una panorámica pormenorizada de los intercambios lingüísticos y culturales que se han producido en la frontera peninsular hispano-portuguesa, y más específicamente en la franja geográfica que va desde Rio de Onor (Portugal)/Rihonor (España) hasta Vila Real de Santo António (Portugal) y Ayamonte (España). Este estudio, que también recoge los intercambios que se están desarrollando en el momento presente, se centra en multitud de aspectos, de entre los que se destacan los siguientes: fuentes de información, proyectos, programas, asociaciones, publicaciones, investigación y especialistas en las áreas que conforman el citado espacio físico -su geografía y su historia, su literatura oral y tradicional, las lenguas y sus contactos, su antropología y su etnografía, su cultura-. Todo con ello con el objetivo de que esta documentación bibliográfica sea una exposición de los trabajos más destacados que han ido viendo la luz, en tiempos pasados y en tiempos recientes, con el fin de se convierta en impulso para futuras investigaciones.
\end{abstract}

\section{Palabras Clave}

Frontera hispano-portuguesa; lenguas en contacto; relaciones entre Portugal y España; relaciones transfronterizas

\begin{abstract}
Through this work, we present a detailed overview of the linguistic and cultural exchanges that have taken place on the Spanish-Portuguese peninsular border, and more specifically in the geographical strip that foes from Rio de Onor (Portugal) / Rihonor (Spain) to Vila Real de Santo António (Portugal) and Ayamonte (Huelva). This study, which also includes the exchanges that are taking place at the present time, focuses on many aspects, among which the following stand out: sources of information, projects, programs, associations, publications, research and specialists in the areas that make up the aforementioned physical space: its geography and its history, its oral and traditional literature, languages and their contacts, their anthropology and ethnography, their culture. All this with the aim that this bibliographic documentation is an exposition of the most outstanding works that have come light, in past times and in recent times, in order to be converted into an impetus for future research.
\end{abstract}




\section{KEYWORDS}

Spanish-Portuguese border; languages in contact; relations between Portugal and Spain; cross-border relations

RECIBIDO 2019-10-14; ACEPTADO 2020-03-22

Trabajo realizado en el ámbito del proyecto Frontera hispano-portuguesa: personas, pueblos y palabras (FRONTESPO-3P), financiado por FEDER/Ministerio de Ciencia, Innovación y Universidades-Agencia Estatal de Innovación, periodo 2019-2022, ref. RTI2018-095899-B-I0, revisión de Navas (2017).

\section{Presentación}

El elevado interés hacia la investigación que desde varias aproximaciones presenta la frontera hispano-portuguesa, lugar de encuentro y de confluencia de lenguas y culturas a lo largo de centenares de siglos, la ausencia de descripciones generales y, paradójicamente, la insuficiencia de trabajos transfronterizos, así como el reconocimiento de que la frontera hispano-portuguesa constituye sin duda un patrimonio inmaterial en peligro de desaparición, nos llevó a trazar un panorama diacrónico y sincrónico sobre los estudios que desde varias perspectivas se han venido desarrollando acerca de la frontera peninsular.

La integración de Portugal y de España en la Comunidad Económica Europea (CEE), en 1986, y en, consecuencia, las ayudas económicas que se han aprobado relativas a los espacios geográficos, al desarrollo de zonas desfavorecidas, a sus costumbres, sus lenguas y sus idiosincrasias, en nuestra opinión, han propiciado los recientes trabajos sobre la frontera hispano-portuguesa, que han proliferado exponencialmente. Unas ayudas europeas que, curiosamente, están concebidas para contribuir a la desactivación del hecho fronterizo, a la neutralización de las fronteras interiores y a la construcción de una nueva Unión Europea (Calderón Vázquez 2015).

Una de las áreas desfavorecidas del espacio peninsular es la conocida como raya ${ }^{1}$, zona que se extiende de oeste a este y de norte a sur en la frontera política entre España y Portugal a lo largo de unos 1250 kms. y que se consolidó, según es sabido, en el Tratado de Lisboa o Tratado de Límites, en 1864. Hasta esa fecha, más que de línea divisoria rígida deberíamos hablar de espacios comunes, de zonas amplias de contacto luso-español (Godinho 2015). No olvidamos -aquí un paréntesis- que, evidentemente, existen otras fronteras entre España y Portugal, como la que se establece en la "ultraperifericidad" de los archipiélagos de Madeira y Azores con las Islas Canarias -la Macaronesia- aunque ahora no contemplemos su análisis bibliográfico.

En esta ocasión, presentamos una panorámica, a todas luces no exhaustiva -no puede encararse esta ingente tarea a título individual- $\mathrm{y}$ avanzamos en las posibles fuentes de información, esen-

1 Véase la descripción de la frontera hispano-portuguesa en Cordero Torres (1960). Texto clásico es el de Pintado y Barrenechea (1972), y más recientes son, entre otros, los de Alonso de la Torre (2006) o, el más divulgativo de Martínez Reñones (2011). 
cialmente en la raya que discurre de norte a sur de la Península Ibérica, posponiendo para futuras entregas la frontera este-oeste ${ }^{2}$. Mostramos aquí las fuentes y recursos relativos a investigaciones, publicaciones, tesis, programas y proyectos, que desde varios puntos de vista -lingüístico, literario, histórico, antropológico, etnográfico, geográfico o cultural-, se han venido realizando o están en curso.

La frontera hispano-portuguesa es una zona despoblada y en recesión. Acabada la agricultura y el contrabando ${ }^{3}$, que eran la base de la economía de la raya, la emigración de sus poblaciones ha convertido este espacio en un conjunto de centros patrimoniales, lugares de atracción turística para los urbanitas, en los que "regionautas" (Godinho 2015) exploran y explotan la herencia que permanece todavía en estas áreas, "fetichizándola". Hubo oficios comunes, se mantuvo la trashumancia y, en parte, se mantiene, a veces, a través de los ríos (como en Cheles, Badajoz). La frontera hispanoportuguesa, desvinculada de sus contextos cotidianos, ha dejado de ser de utilidad para ser de futilidad, en palabras de Godinho (2015), reducto de monumentos pero no de memoria.

Dicha frontera hispano-portuguesa, además de una raya seca, tiene también otra húmeda. Habrá que considerar los flujos de pescadores entre ambas márgenes de los ríos Miño, Duero, Tajo y Guadiana: a los portugueses, marineros y obreros de las industrias conserveras, emigrados, de forma eventual o permanente, a poblaciones españolas; y a los españoles, enrolados en barcos portugueses y, como consecuencia, un conjunto de términos propios del mar prestados, según los casos, de una a otra lengua (Cáceres y Corbacho 2013).

\section{Proyectos europeos para desactivar la frontera a nivel autonómico}

Son diversos los proyectos que a lo largo de los últimos años se han venido desarrollando en colaboración con organismos oficiales y particulares con el apoyo de la Unión Europea con el objetivo de la "desactivación fronteriza" (Calderón Vázquez 2015). Por ejemplo, la Cooperación Territorial Europea, Interreg, en su Política de Cohesión Europea para intensificar la relación internacional transfronteriza entre regiones localizadas en las fronteras internas y externas de la Unión Europea, de manera que se genere un espacio único "de movilidad de personas, bienes y capital [donde las fronteras] tienen que dejar de ser zonas de separación para convertirse en franjas de unión” (Heredero de Pablos y Olmedillas Blanco 2009: 212-213)4. Así mismo, en esa

2 Para aspectos comunes de tipo antropológico entre Galicia y Portugal, véanse los trabajos de referencia de Godinho. Para aspectos de tipo cultural, etnográfico y musicales, se deben consultar las publicaciones de Proyectos como O Celtismo e as suas repercussões na música na Galiza e no norte de Portugal, coordinado por Salwa El-Shawan Castelo-Branco de la Universidade Nova de Lisboa, con el apoyo financiero de la Fundação para a Ciência e a Tecnologia, 2009-2013, así como la Acción Integrada Luso-Española del mismo título (2010-2012), de Salwa El-Shawan Castelo-Branco y Susana Moreno Fernández (Universidade Nova de Lisboa), Jorge Freitas Branco (Instituto Universitário de Lisboa), (coords.), Jorge Freitas Branco (Instituto Universitário de Lisboa), Enrique Cámara de Landa (Universidade de Vigo) y Luis Costa VázquezMariño, Grazia Tuzi y Eduardo Contreras Rodríguez (Universidad de Valladolid), miembros del equipo.

3 El contrabando, claro está, no ha desaparecido y se centra en otros productos, ganado, tabaco y drogas. Sigue otros esquemas y tiene otros actores.

$4 \quad$ No está de más recordar en estos días recientes de llegada masiva de inmigrantes y en los actuales, donde estamos confinados por la pandemia que afecta a todo el mundo, que las fronteras existen, lo que puede desaparecer son los controles que, cuando los gobiernos consideran conveniente, restablecen, como es el caso. 
dirección van las propuestas de los respectivos gabinetes de Iniciativas Transfronterizas como, por ejemplo, el de Extremadura 5 .

Como resultado de las iniciativas europeas se han ido produciendo a lo largo de los años, encuentros y publicaciones que manifiestan el interés de los estudiosos por los temas fronterizos. Algunos han partido de instituciones autonómicas españolas, es el caso de Expresiones simbólicas y cultura de fronteras en la raya de Portugal (1993) (Hernández León y Castaño Madroñal 1992-1993) o la Revista del Centro de Estudios Andaluces (ambos dependientes de la Consejería de Cultura de la Junta de Andalucía), o el Plan Portugal (2009, retomado en 2016) de la Junta de Extremadura. Otras iniciativas han surgido de organismos portugueses, como el proyecto Além da Água, comisionado por Jorge Castanho y Mar Villaespesa, en 1995. En ese sentido y, con motivo de la construcción del embalse de Alqueva, teniendo como hilo conductor el río Guadiana, este proyecto se ocupó, especialmente, de las relaciones económicas, agrícolas, sociales, ecológicas, hidrográficas o políticas entre el Alentejo y Extremadura y Andalucía. Resultado de todo ello fue una serie de actividades, performances e instalaciones que cubrieron un amplio espectro geográfico, en las que participaron desde artistas, arqueólogos e ingenieros hasta geógrafos, historiadores o lingüistas, pasando por filósofos, matemáticos o, incluso, surfistas. De las varias realizaciones conseguidas consta, entre otras, una Exposición/Taller Além da Água. Copiacabana en el Museo Extremeño e Iberoamericano de Arte Contemporáneo de Badajoz, en 1996, y la publicación de los documentos que muestran parte del trabajo llevado a cabo (Camacho et al. 1999).

Otras iniciativas son de un ámbito más extenso, como el citado Programa InTerreg. Así el INTERREG I (1989-1993), destinado a estructuras transfronterizas específicas; el INTERREG II (1994-1999), referido, entre otros, al espacio de Andalucía con el Algarve y Alentejo, Proyecto de inventario de patrimonio etnográfico de la zona transfronteriza con Portugal (2006), con frutos en diversas publicaciones, principalmente, a cargo de los investigadores Elodia Hernández León, Ángeles Castaño Madroñal, Victoria Quintero y Rafael Cáceres; el INTERREg III (2000-2006), Relaciones lingüísticas y literarias entre España y Portugal desde los inicios del siglo XIX hasta la actualidad, a partir del cual se establecieron lazos entre universidades portuguesas (Beira interior) y españolas (Salamanca), se celebraron dos congresos (Évora 2006 y Salamanca 2007) y se publicaron las respectivas actas (Marcos de Dios (Ed.) 2007); el INTERREG IV (2007-2013), Innovación $y$ del conocimiento, medio ambiente y prevención de riesgos; o el INTERREG V (2014-2020), Impulso a la investigación y a la innovación transfronteriza entre España y Portugal.

Algunas ayudas procedentes de la Comisión Europea, como el Fondo Europeo de Desarrollo Regional, FEDER, están orientadas a regiones con problemas definidos por la citada Comisión, relativos al empleo, al mercado laboral o a la periferia específica de cada comunidad, entre otros. En ese sentido la Associação de Defesa do Património de Mértola (Beja) (ADPM) puso en marcha un proyecto, entre 2007-2013, Tradições Orais do Baixo Alentejo: Memórias Orais e Aprendizagens Globais, con el apoyo de los municipios del distrito de Beja, Almodóvar, Barrancos y Mértola. Se recogieron testimonios grabados y filmados sobre plantas aromáticas y medicinales en Mértola, el oficio de zapatero en Almodóvar y el contrabando, entre otros asuntos, en Barrancos, en el libro bilingüe y respectivo CD-Rom, coordinados por Revez y Cascalheira (Coords.) (2010), Do saber ao contar/Del saber al contar.

$5 \quad$ Véase información general en su página gitextremadura.gobex.es. Sobre algunas publicaciones de ese organismo sobre “a fala”, pueden consultarse, por ejemplo, Gargallo Gil (1999), Martín Galindo (1999) o Rey Yelmo (1999). 
En 2007, la Comisión Europea aprobó el Programa Operativo de Cooperación Transfronteriza Portugal-España (2007-2013) (POCTEP) para el desarrollo, entre otros, de recursos del patrimonio histórico y cultural (Calderón Vázquez 2015). Dicho Programa dividió la frontera de los dos países en diecisiete Unidades Territoriales (NUTs), de ellas diez en Portugal y siete en España, distribuidas en cinco espacios geográficos: Región Norte-Comunidad Autónoma de Galicia; Región Norte-Comunidad Autónoma de Castilla y León; Región Centro-Comunidad Autónoma de Extremadura-Región de Alentejo; Región de Alentejo-Comunidad Autónoma de Andalucía-Región de Algarve ${ }^{6}$. Ahí, en estas áreas concretas, nos parece que debería incidir la recogida bibliográfica de la frontera hispano-portuguesa, la búsqueda de temas que contemplen los asuntos que interesan a la referida raya en todos los ámbitos posibles incluido, claro está, el lingüístico.

Posteriormente, en 2009, se crearon dos eurorregiones, una comunidad de trabajo, Euro-Ace, entre los distritos portugueses de Alentejo - Centro y la región de Extremadura. Recordemos que Badajoz y Cáceres mantienen grandes intereses con Portugal, pues a este lado de la frontera, por ejemplo, se enseña la lengua portuguesa en 88 centros. En dicha eurorregión se llevan a cabo muchas iniciativas económicas, sanitarias, sociales, empresariales, deportivas, medioambientales, de prevención de riesgos, turismo, entre las más significativas ${ }^{7}$. La otra es la Agrupación Europea de Cooperación Territorial (AECT) entre Zamora y Salamanca y Trás-os-Montes y el Duero superior. En esta última se privilegian aspectos de competitividad empresarial, transporte público, empleo o desarrollo sostenible.

Los proyectos más innovadores sobre la Cooperación Transfronteriza Institucional son los referidos a las Eurociudades, pensadas con el objetivo de crear un nuevo modelo donde sus ciudadanos tengan una Tarjeta de Eurociudadanía que les permita acceder a una serie de servicios, polos de desarrollo únicos, promoción turística compartida, celebraciones gemelas o ventanilla única transfronteriza. En este momento están creadas cinco. La pionera fue la Eurociudad de Chaves-Verín (2007), seguida de la de Tui-Valença do Minho (2012), Ayamonte-Vila Real de Santo António-Castro Marim (2013), Badajoz-Elvas-Campo Maior (2013) y, la última, MonçãoSalvaterra de Miño (2015). La de Chaves-Verín, se ha especializado en la Eurociudad del Agua porque presenta la mayor concentración de aguas termales y manantiales de la Península. La de Tui-Valença do Minho tiene un parque de bomberos voluntarios, un teatro y una piscina, que administran conjuntamente. La de Monção-Salvaterra de Miño ha conseguido un descuento del $10 \%$ en actividades de ocio, como piscina, espectáculos o deportes, gracias a una tarjeta común. La de Badajoz-Elvas-Campo Maior pretende fomentar el bilingüismo, el intercambio de colegios y una propuesta turística conjunta, EUROBEC. Todas estas iniciativas surgidas gracias a los fondos procedentes de la Unión Europea, como los FEDER o INTERREG V-A.

\footnotetext{
6 Pueden consultarse algunos resultados de los convenios entre estas tres regiones en 15 años (2000-2014) de Cooperación Transfronteriza entre Alentejo, Algarve y Andalucía en www.juntadeandalucia.es/export/drupajda/15_axos_ CTF.pdf. Existe, asimismo, un Gabinete de Iniciativas Transfronterizas (GIT) con sus ediciones respectivas.
}

7 Véase a este respecto la memoria descriptiva de 2018 https//www.euro-ace.eu/sites/default/files/anuario_ euroace_2018.pdf [consulta 28 de junio de 2019]. 


\section{Proyectos propiamente ibéricos}

Algunas poblaciones, independientemente de estar enmarcadas en estas iniciativas europeas organizadas desde arriba, toman sus propias providencias desde abajo creando acuerdos mixtos que permiten desarrollar sus tareas conjuntamente, como es el caso del Protocolo de Cooperación Transfronteriza entre los Bomberos de Mourão (Alentejo) y Villanueva del Fresno (Badajoz) o, entre los mismos profesionales de Barrancos y el Consorcio Provincial de Huelva; el Consorcio Transfronterizo de Ciudades Amuralladas Ciudad Rodrigo y Almeida ${ }^{8}$; o el Movimento Cívico DART (Defender, Autonomizar e Rejuvenescer Trás-os-Montes e Alto Douro juntamente con la región autonómica de Castilla y León) que ha creado la plataforma Rede Ibérica Ocidental para una Nova Ordenação Raiana (RIONOR).

\section{Iniciativas universitarias portuguesas y españolas}

Para llevar a cabo la tarea acerca de los estudios sobre la frontera hispano-portuguesa hay que estar, asimismo, atentos a las posibles iniciativas en forma de Encuentros, Simposios, Jornadas, etcétera, o publicaciones de las universidades. Por ejemplo, la Universidad de León tiene una página dedicada a Frontera. La Raya Ibérica9 ; la Universidad Carlos III de Madrid dedicó en su Revista de Historiografía, en 2015, editado por Jacobo García Álvarez, un número sobre la frontera en cuestión; mientras que la Universidad de Barcelona, en 2013, en su Facultad de Filología, organizó un encuentro de Poesia a la frontera donde se contemplaban diferentes situaciones fronterizas. En 2011, se realizó el Congreso Fronteras Ibéricas, en la Facultad de Ciencias Políticas y Sociología de la Universidad Complutense de Madrid, organizado por el grupo de investigación, El discurso geopolítico en las fronteras en la construcción socio-política de las identidades nacionales: el caso de la frontera hispano-portuguesa en los siglos XIX y XX (2007-2011), cuyo investigador principal fue Heriberto Cairo Carou. De esta misma universidad y coordinado por el mismo investigador, es asimismo el proyecto Cooperación transfronteriza y (des)fronterización: actores y discursos geopoliticos transnacionales en la frontera hispano-portuguesa (2008-2016), financiado por el Ministerio de Economía y Competitividad (VI Plan Nacional de Investigación Científica, Desarrollo e Innovación Tecnológica). Este autor y su equipo han venido trabajando regularmente en la frontera hispano-portuguesa desde el punto de vista sociológico, político y antropológico. La Universidad de Huelva, con apoyo de los ayuntamientos de Ayamonte y de Vila Real de Santo Antonio (Faro), organizó fronteiras ${ }^{10}$. I Seminario Hispano-Luso. Patrimonio Industrial Conservero y Salazonero, Ayamonte, 15-19 de julio de 2015. En la Universidad de Extremadura, por su parte, tuvo lugar antes, en 1994, el Congreso Internacional Luso-Español de la Lengua y Cultura en la Frontera con amplia representación temática cuyos resultados se

8 Para fortificaciones que recorren la frontera puede verse Cayetano Rosado (2018).

9 Disponible en http://ulefhp.unileon.es/publicaciones.htm [consulta 8 de sept., 2016]).

10 El Proyecto cátedra fronteiras, 2015, promovido por el Ayuntamiento de Santarém y las universidades Lusíada de Lisboa, Lusíada do Porto y la de Sevilla, y la Asociación Ibérica para la Inovação, Património, Arquitectura e Cultura (AIPAC), desvela, investiga y difunde las realidades comunes entre España y Portugal con especial incidencia en la arquitectura y cultura. 
publicaron en 1996, coordinados por Juan M. Carrasco González y Antonio Viudas Camarasa. En la misma universidad extremeña, dentro del área de Filología Portuguesa se ha fundado por profesores de la Facultad de Filosofía y Letras una Sociedad Extremeña de Estudios Portugueses y de la Lusofonía (sEePLu), que estudia la cultura, la lengua y el arte en los países de expresión portuguesa. Dicha Sociedad, además, de editar anualmente la revista Limite, organiza bianualmente congresos temáticos.

La Universidad de Évora, juntamente con la de Extremadura y el Ayuntamiento de Barrancos firmaron un protocolo para Estudar, preservar e valorizar as falas dos povos de Cedillo, Herrera de Alcántara e Barrancos (2008-2014). La misma universidad portuguesa de Évora puso en marcha un Mestrado em Estudos Ibéricos, con resultados de trabajos específicos de investigación, uno sobre literatura oral y tradicional en tierras de frontera (Quijada 2012), otro, en curso, sobre la normalización de una lengua ágrafa de frontera, el barranqueño (Correia s.d.) ${ }^{11}$. También en la misma universidad del Alentejo, Évora, tienen lugar encuentros regulares con especialistas como la I Jornada de falares fronteiriços (febrero, 2008); o el posterior Seminario internacional O oceano Atlântico: espaço de intercâmbios culturais e linguísticos (13-14 septiembre, 2016). No hay que descuidar, asimismo, las investigaciones amparadas por la Universidade de Trás-os-Montes e Alto Douro, como la publicación, con la Escuela Oficial de Idiomas de Valladolid, de la revista Nós -otros y la organización en octubre de 2016, de un Colóquio ibérico de estudos rurais. Hay que tener presente la Universidade da Beira Interior, que preparó el año 2016 el I Encontro de línguas ibéricas. Otros organismos docentes como el Centro de Línguas, Literaturas e Culturas da Universidade de Aveiro, con el apoyo del ayuntamiento de Miranda do Douro, puso en marcha el Colóquio internacional sobre ecolinguísmo e línguas minoritárias. Uma homenagem a Amadeu Ferreira (15-18 junio, 2016, Aveiro y Miranda do Douro) y, en mayo de 2018, organizó el I Congresso internacional em variação linguística nas línguas românicas. Más recientemente, en 2018, en la Universidade Nova de Lisboa, Paula Godinho, Dulce Simões y Maria Alice Samarra, organizaron un Encontro internacional fronteiras e topografias do poder: culturas da raia e economias possíveis, entre discursos e práticas.

\section{Iniciativas de proyectos de investigación a partir de Planes Nacionales portugueses y españoles}

Es preciso tener en cuenta, además de los ya citados de las varias universidades españolas y portuguesas, proyectos enmarcados en los diferentes Planes Nacionales, I+D e I+D+i, que el Ministerio de Educación y Ciencia de España y el de Ciencia e Innovación, y sus homólogos de Portugal, han ido aprobando. Citamos algunos: El capital social a ambos lados de la frontera: aplicación de un modelo de indicadores para el estudio del capital social y los cambios sobre las percepciones en la Raya de Extremadura, referido a la Sierra de Gata (Cáceres-España) y la Beira Interior Sur (Región Centro de Portugal) (2008), del Grupo de Estudios Sociales Aplicados de la Facultad de Formación del Profesorado de la Universidad de Extremadura, Eusebio Medina García investigador principal (http://www.gessa-ex.es). El proyecto de investigación más reciente y completo que abarca 
la situación cultural, lingüística y etnográfica de la frontera hispano-portuguesa es, sin duda FRONTESPO-3P, RTI2018-095899-B-100, investigador principal Xosé Afonso Álvarez, Frontera hispano-portuguesa: Personas, pueblos y palabras (2019-2022), financiado por FEDER/Ministerio de Ciencia, Innovación y Universidades-Agencia Estatal de Innovación, que es continuación de otro anterior, Frontera hispano-portuguesa: Documentación lingüística y bibliográfica (FRONTESPO 2015-2017), Programa Estatal de Investigación, Desarrollo e Innovación Orientada a los Retos de la Sociedad, Ministerio de Economía y Competitividad (http://www.frontespo.org/). Dentro de su página en internet, además de fuentes bibliográficas, enlaces, blogs, grabaciones en vídeo, entre otros, se encuentra el Tesoro léxico de la frontera hispano-portuguesa realizado por José Antonio González Salgado (http://www.frontespo.org/es/tesoro).

\section{La investigación en centros ajenos a la universidad}

Conviene revisar los proyectos de los Centros de Investigación portugueses, como los del Centro de Linguística da Universidade de Lisboa, CoRdiAL-sin: Corpus dialectal para o estudo da syntaxe, Ana Maria Martins (Coord.) (disponible en http://www.clul.ul.pt/pt/investigacao/212-cordial-sin-syntax-oreinted-corpus-of-portuguese-dialects); o el Tesouro dialectal português (TEDIPOR), del mismo Centro y del Centro de Estudos de Linguística Geral e Aplicada da Universidade de Coimbra (CELGA), coordinado en 2008 por Clarinda Azevedo Maia (disponible en limiar.clul. ul.pt/posters/13_alvarez_maia.pdf); así como los del Consejo Superior de Investigaciones Científicas, por ejemplo, la publicación en red del Atlas Lingüístico de la Península Ibérica (ALPI) ${ }^{12}$. O la consulta a las bases de datos del Corpus oral y sonoro del español rural (COsER), Universidad Autónoma de Madrid, Universidad Carlos III de Madrid y Université de Montréal ${ }^{13}$, dirigido por Inés Fernández-Ordóñez (un buen recurso para trabajos relacionados con la frontera, como por ejemplo, el de Serradilla Castaño 2006); así como el proyecto, Catálogo de Voces Hispánicas del Centro Virtual Cervantes, dirigido por Francisco Moreno Fernández, de la Universidad de Alcalá de Henares, que incluirá material sonoro también de los territorios de la frontera (http://cvc. cervantes.es/lengua/voces_hispanicas/. Pero también hay que mencionar otros focos de investigación, no siempre bien conocidos, como el Centro Interdisciplinar de Documentação Linguística e Social (CIDLes), en Minde (Alcanena, Santarém), dedicado a la documentación y conservación del patrimonio cultural en Europa, donde se trabaja en la normalización y normativización del minderico (Ferreira et al. 2015) pero también en las lenguas minoritarias y amenazadas.

\section{Otras iniciativas relativas a la frontera}

Hay que seguir las revistas de la especialidad en la amplia área de la raya, pero también las publicaciones del Centro de Estudos Ibéricos (CEI) de Guarda, fundado por Eduardo Lourenço, en 1999, que viene organizando cursos de verano y seminarios cuyos textos salen a la luz regularmente,

12 Parte de sus datos ya disponibles en http://alpi.csic.es.

13 Disponible en http://www.lllf.uam.es/coser/index.php. 
como los editados recientemente por Jacinto y Cabero $(2014)^{14}$. No podemos dejar a un lado la importancia que tienen las fundaciones a la hora de organizar jornadas, exposiciones o publicaciones que tengan como objetivo la frontera. En ese sentido, es muy dinámica la Fundación Hispano-Portuguesa Rei Afonso Henriques (FRAH), que contempla una Agenda Cultural Transfronteriza Zamora-Bragança, donde se incluyen también temas empresariales.

\section{Trabajos Final de Grado, Trabajos Fin de Máster y Tesis Doctorales en universidades portuguesas y españolas}

Son imprescindibles los vaciados de los TFG, TFM y las tesis doctorales que sobre la frontera se van realizando, cuya información no es siempre detectable debido a las inesperadas temáticas de área de conocimiento, a veces, alejadas del campo de consulta de las Humanidades. Veamos, por ejemplo, las que se han venido defendiendo en el Instituto Superior de Ciências do Trabalho e da Empresa, de Lisboa entre otros (Silva 1999), en la especialidad en Sociología (Lopes 2001) o en Historia Contemporánea (Pires 1997). De la misma manera, interesa ver los trabajos que se van realizando en la Escuela Superior de Educación del Instituto Superior Politécnico de Viseu. Es conveniente, por otro lado, revisar las investigaciones llevadas a cabo en la Universidade de Évora en Sociología (Domingues 2003), en la Universidade Nova de Lisboa en Antropología (Bastos et al. 1982), en la Universidade de Lisboa en Historia Moderna (Candeias 1998), o en la Universidade Moderna de Beja en Antroposociología (Domingues 1995). Se deben consultar, asimismo, los trabajos de investigación que se realizan en la Universidade do Algarve, como el dedicado al contrabando (Rodrigues 2013) o en las universidades españolas como la de Extremadura donde, en 2015, se leyó la tesis doctoral de Silvia Amador Moreno, Español y portugués en contacto. Las actitudes lingüísticas en zona fronteriza: Extremadura y Alentejo.

\section{Proyectos fronterizos a cargo de los centros administrativos de poder españoles y portugueses}

No olvidemos la tarea divulgativa, pero también científica, que realizan las diputaciones españolas, como la de Badajoz, que edita, entre otros títulos, O Pelourinho. Boletín de Relaciones Transfronterizas, que incluye especialmente asuntos relativos al patrimonio. O las equivalentes de los distritos portugueses. Conviene, asimismo, revisar las diferentes asociaciones que propugnan la defensa de sus comunidades. En España, por ejemplo, existe la Asociación Estudio y divulgación del patrimonio lingüístico extremeño (APLEX), que está desarrollando el Archivo sonoro de las hablas extremeñas (ASHE); Asociación A Nosa Fala, que promueve las tres variantes, lagarteiru, mañegu y valverdeiro, hablas, respectivamente, de Eljas, San Martín de Trevejo y Valverde del Fresno (Cáceres) (información disponible en http://www.anosafala.com/sobri-a-asociacion); Asociación U Lagartu Verdi, que edita la revista Anduriña, escrita en la variante lagarteira. Así como, por ejemplo, en Portugal, la Associação Cultural Além-Guadiana, creada en 2008, en defensa de la cultura oliventina. 
Hay que estar atentos a congresos, simposios, encuentros o jornadas de tipo local, en ocasiones, difíciles de detectar. Más conocidos son los que se encuentran ya en bases de datos, como las Jornadas ibéricas de investigadores en ciencias humanas y sociales. Encuentros/Encontros de Ajuda (celebradas en Olivenza, 1985), cuyas actas se publicaron dos años más tarde. U otras más recientes, como el Congreso internacional APLEX 2004. Patrimonio lingüístico extremeño, celebrado en Cáceres; las Jornadas de historia en Llerena (Lorenzana de la Puente y Mateos Arcacíbar, coords., 2008) ${ }^{15}$; la International conference on endangered languages in Europe (2013, organizada por V. Ferreira et al.), así como el I Simpósio internacional sobre línguas e variedades linguísticas ameaçadas na Península Ibérica: Documentação e revitalização - LAPI 2016, ambos en Alcanena, organismo que realiza, regularmente, encuentros como este, o colabora con otros, como el llevado a cabo en Coimbra en septiembre de 2017, New Speakers in a Multilingual Europe o el más reciente, celebrado en Lisboa en 2019, Línguas e variedades ameaçadas na Península Ibérica. Así como las regulares Jornadas del Patrimonio de la Sierra de Huelva, que celebra la Federación de las Asociaciones de la Sierra de Huelva, y que tienen lugar en el amplio espectro de las comunidades de la citada Sierra de Huelva, muchas de ellas relacionadas con Portugal; la colección La provincia de Huelva. Historia de sus villas y ciudades, que edita la Diputación de Huelva, así como Huelva en su historia (López Martínez 2004); o las que edita la propia Universidad de Huelva como Realidad social en Andalucía, Algarve y Alentejo (Gualda Caballero, dir., 2008) ${ }^{16}$. Las Jornadas de Historia de Ayamonte, que ya han cumplido veinte ediciones; o las iniciativas de los Ayuntamientos de Aroche, Encinasola -Jornadas de Encuentro entre Marochos y Barranqueños (Oliver 2005)- Moura o Barrancos ${ }^{17}$.

\section{Los museos como lugar de encuentro intrafronterizos}

Conviene mencionar los museos que en los últimos años han proliferado y que pueden editar, o están publicando, monografías locales. En ese sentido, no hay que olvidar la tarea que, en España, desarrolla el Museo Extremeño e Iberoamericano de Arte Contemporáneo de Badajoz (meiAc), al estrechar lazos a ambos lados de la frontera, o el Centro de Interpretación del Contrabando, en Vilardevós (Ourense), el Museo Etnológico, en Puerto Seguro (Salamanca), el Centro de Interpretación de la Frontera, en Oliva de la Frontera (Badajoz); o en Portugal el Museu do Contrabando de Santana de Cambas (Mértola); el Centro de Ciência do Café, en Campo Maior (Portalegre), con salas también dedicadas al contrabando; Espaço, Memória e Fronteira. Museu do Contrabando e da Emigração Clandestina, en Melgaço (Viana do Castelo); Museu Etnográfico con alusiones al contrabando, en Vilar de Perdizes (Montalegre); el Museu Municipal de Arqueologia e Etnografia, en Barrancos (Beja) o, por decir alguno más, el Museu Ibérico da Máscara e do Traje, en Bragança.

\footnotetext{
15 Disponible en file://C:/Users/Usuario/Downloads/Dialnet-IberismoLasRelacionesEntreEspanaYPortugalHistoriaY-329934.pdf.

16 Disponible en https://estrellagualda.es/descargas/publicaciones/2008_Realidad_Social_AAA.pdf.

17 Veánse, en ese sentido, por ejemplo, las Actas de las I Jornadas Transfronterizas sobre la Contienda HispanoPortuguesa (1996).
} 


\section{La prensa local, fuente de información}

Los periódicos y revistas editados en las comunidades limítrofes son un buen medio para recoger las actividades, ferias, creaciones e investigaciones de los correspondientes lugares. Ya hemos citado Nós-otros, demos otros ejemplos como El Correo de Zamora (Zamora), Aquí (Badajoz), Jornal da Raia ${ }^{18}$, El Filandar/O Fiadeiro, publicación Ibérica de Antropologia e Culturas Populares (Bajo Duero) ${ }^{19}$, que acoge, entre otros, temas portugueses, castellanos o mirandeses; Limite. Revista de Estudios Portugueses y de la Lusofonía, de la Universidad de Extremadura; o Estudios Fronterizos, de la Universidad de Málaga, donde se encuentran asuntos sobre la frontera que nos ocupa. Recordemos también revistas de estudios transnacionales, algunas ya desaparecidas como Boca Bilingue/Boca Bilingüe, Espacio/Espaço y la reciente Suroeste, editada por la Junta de Extremadura.

\section{Internet, la radio, la televisión y la prensa}

Dejar a un lado las redes sociales, las emisoras de radio y los programas de televisión sería, evidentemente, un lapsus imperdonable. Tanto unas como otros son fuente imprescindible de informaciones intrafronterizas. En youtube, por ejemplo, Rayanos Magazine (rayanos.com) da cuenta de las actividades que tienen lugar en esas tierras de frontera como el Festival de Música o de cine organizado por Marvão y Valencia de Alcántara. Las televisiones autonómicas dedican espacio a las áreas citadas, así, por ejemplo, el Canal Extremadura ha realizado varios programas como el dedicado a Barrancos, De pueblo en pueblo ${ }^{20}$; pero también la generalista, Radio Televisión Española, ha dedicado alguno como Comando actualidad, que ha titulado Un pie en España y otro en Portugal $^{21}$, centrado en La Tojera (Badajoz). Lo mismo sucede con radio Extremadura Lusitânia Express, con una programación bilingüe relativa a intereses de la frontera. Por último, recientemente, El País de tirada nacional, dedicó un artículo, a página completa, a la raya, firmado por Juan Antonio Aunión (30/5/2019) lo que viene a completar el interés que parece que existe por esta zona común a los dos países.

\section{Bibliografías lingüísticas sobre la frontera hispano-portuguesa}

Las poblaciones cercanas a la raya, tanto portuguesas como españolas, no han sido objeto de estudios abundantes a lo largo de los años. Bien es cierto que desde el ingreso en la Unión Europea ha habido, en la década de los años 80 , ya se ha dicho, un interés cada vez mayor por las minorías lingüísticas y, claro está, por estas poblaciones. Así se elaboró la Carta Europea de las Lenguas Regionales y Minoritarias, gracias a la cual la Comisión Europea empezó a aplicar una serie de recursos

18 Disponible en www.noticiascyl.com.

19 Disponible en http://bajoduero.org/el-filandar/.

20 Disponible en http:/www.canalextremadura.es/alacarta/tv/videos/de-pueblo-en-pueblo-170915)

21 Disponible en http://www.rtve.es/alacarta/videos/comando-actualidad/comando-frontera-portugal/884815/. 
para apoyar a dichas comunidades. Es tal vez el momento de recordar la Asociación Internacional para la Defensa de las Lenguas y Culturas Amenazadas (AIDLCM), cuyo acto fundacional tuvo lugar en 1962 y entre cuyos objetivos se incluye, como indica su denominación, la defensa y promoción de las lenguas y culturas amenazadas por la decadencia, degradación o extinción a causa de la discriminación que sufren en su país (Nagore y Puig, eds., 1999: 11). Pero a partir de los años 90 los investigadores se han ido interesando en recoger testimonios lingüísticos, literarios, antropológicos, sociológicos, etnográficos, entre otros, de manera que queden evidencias de un tiempo ya casi desaparecido. Generalmente han sido autores peninsulares los que han contribuido con sus trabajos a la investigación de las variedades autóctonas, si exceptuamos los investigadores extranjeros del siglo pasado, como Oskar Frink, Fritz Krüger, Helmut Lüdtker o Göran Hammarström. Sin embargo, recientemente, estudiosos americanos -Clements y Lorenzino (2006) -, búlgaros -Irena Stefanova (2000) -, o alemanes Christina Ossenkop (2010) - algunos procedentes del ámbito de la criollística, se han aproximado a esta realidad peninsular.

En 1998, elaboramos una sucinta aproximación a los estudios lingüísticos que encaraban la raya como punto de partida (Navas 1998), ampliada posteriormente (Navas 1999), aproximación que, posteriormente, completamos con la colaboración al proyecto bibliográfico coordinado por Manuela Barros Ferreira, Língua e história na fronteira Norte-Sul (2006) -más tarde revisado con el título Língua e cultura na fronteira Norte-Sul (2010) -, ambos bajo el paraguas del Campo Arqueológico de Mértola. En la primitiva iniciativa referida al citado proyecto de 2006 colaboraron estudiosos del ámbito de la lingüística, de la historia, de la cultura, tanto de España (X. Henrique Costas, J. A. González Salgado, M. V. Navas Sánchez-Élez y A. Viudas Camarasa) como de Portugal (E. Carrilho, M. Barros Ferreira, A. Ferreira, E. Ramos y M. Rego). En la revisión posterior de 2010, que presenta más de 6000 títulos, participaron, además, por parte portuguesa, Fátima Palma y Gustavo Duarte. El propósito de esta investigación fue el de poner a disposición una serie de títulos que abarcasen «desde o extremo Nordeste de Portugal ao extremo sul, ou seja de Bragança -Oeste de Zamora até ao Oriente algarvio- [...] onde se encontrassem os dados indispensáveis para o conhecimento dessas linguagens [Portugal y España], da cultura envolvente e das suas origens» (Ferreira 2006: 3). Porque la historia, la cultura y la lengua de los pueblos están estrechamente unidas se pensó en abarcar estos tres campos para las cinco zonas en las que se dividió la frontera de norte a sur (no de este a oeste, en la frontera del río Miño, insistimos de nuevo). La zona I portuguesa de Bragança se correspondió con la española de Zamora. La zona II, este del distrito de Guarda, con el oeste de la provincia de Salamanca. La zona III comprendía los distritos portugueses de Castelo Branco y de Portalegre y la provincia española de Cáceres. El distrito de Évora, zona Iv, con la provincia de Badajoz. Y por último, en la zona v estaban los distritos de Beja y Faro y la provincia de Huelva. Por último, los títulos, que se incluyeron en dicho proyecto, abarcaban el espacio geográfico comprendido entre la Vía de la Plata española, excepto en la parte sur, y la carretera IP 2 portuguesa: en total, alrededor de unos $30 \mathrm{kms}$. a ambos lados de la frontera política.

El proyecto en marcha más reciente, según nuestros datos, es el ya referido anteriormente, Frontera Hispano-Portuguesa: Personas, Pueblos y Palabras (2019-2022) (FrontesPo-3P), www.frontespo.org. Entre sus objetivos se encuentra crear una Bibliografía multidisciplinar de la frontera hispano-portuguesa, comprendiendo también la frontera gallego-portuguesa, en la que ya se pueden localizar cerca de 1500 registros en línea a los que hay que sumar unos 5000 en las últimas fa- 
ses de edición. En su página virtual se encuentran interesantes enlaces referidos a la citada raya de organismos oficiales y de grupos particulares. Se pretende en su desarrollo recoger un corpus oral, grabado también en vídeo, en diversas localidades a ambos lados de la frontera, en un área geográfica de $15 \mathrm{kms}$. comprendida a ambos lados de la misma, espacio a su vez dividido en diez zonas, con cuatro localidades, dos portuguesas y dos españolas. Durante su ejecución, en la primera fase de FRONTESPO (2015-2017), se han explorado 57 localidades españolas y portuguesas, en las que se han realizado 217 entrevistas individuales o grupales a 237 informantes distintos en 64 puntos. En total, el corpus supera las 290 horas en bruto de grabación en vídeo, a las que hay que sumar 53 horas de las que solo existe grabación de audio, ya que los informantes se negaron a prestar su imagen. Así queda reflejado el mapa de trabajo, la mayor parte ya realizado en 2016: 1. Pontevedra - Viana do Castelo (Sela, municipio de Arbo), Guláns / As Neves - Sobreira, Bemposta, Cova, Cachada, etc.; 2. Ourense -Vila Real; 3. Zamora-Bragança; 4. Salamanca-Guarda (La Bouza- Vale da Mula (Figueira de Castelo Rodrigo), S. Pedro de Rio Seco / Vilar Formoso, Escarigo, Almofala, Vermiosa, Malpartida, etc. (contraparte de La Bouza); 5. Salamanca-Guarda (otras localidades); 6. Cáceres-Castelo Branco; 7. Cáceres-Castelo Branco (otras localidades); 8. Badajoz-Portalegre; 9. Badajoz-Évora-Beja; 10. Huelva-Faro. Los temas incluidos tienen que ver no solo con la lengua sino también con otros campos del saber como economía, geografía, historia o antropología. El interés de esta iniciativa está acompañado por la recuperación de otros elementos, como objetos de la cultura de las comunidades, información etnográfica, actitud lingüística de los hablantes, edición de vocabularios de léxico tradicional; tarea que, una vez finalizada, estará disponible en forma virtual para su consulta en abierto. El equipo de investigadores, encabezado, ya se ha dicho, por Xosé Afonso Álvarez Pérez (Universidad de Alcalá de Henares), comprende especialistas portugueses, Fernando Brissos (CLUL), Vera Ferreira (CIDLes, University of London), y españoles, Juan M. Carrasco González (Universidad de Extremadura), Javier Jairo García Sánchez (Universidad de Alcalá de Henares), José Antonio González Salgado (Campo Arqueológico de Mértola), Ignacio López de Aberasturi Arregi (Universidad de Granada), José Enrique Gargallo (Universidad de Barcelona), María Victoria Navas Sánchez-Élez (Universidad Complutense de Madrid, CLUL), Maria Filomena Gonçalves (Universidade de Évora), José Pedro Ferreira (Instituto de Linguística Teórica e Computacional (ILTEC), Miroslav Valeš (Universidad de Liberec, Chequia) y Víctor Lara Bermejo (Universidad Nacional de Educación a Distancia).

En las fronteras políticas, como es sabido, se suelen producir muchos contactos e intercambios de amplio espectro, también el lingüístico. En el caso de la portuguesa y española tenemos casos de lusismos en andaluz, especialmente en la provincia de Huelva: Encinasola, Puebla de Guzmán, Sanlúcar del Guadiana, San Bartolomé de la Torre, Villablanca, San Silvestre de Guzmán, Lepe o Ayamonte (Campo de Ayamonte: entre otros, La Estacada, Santa Clara, Isla Canela, Punta del Moral, El Romerano, Puerto Carbón, Puerto la Laja, El Espartero, El Rocín). Por cierto, en la zona próxima del río Guadiana se puede incluso confirmar un bilingüismo en retroceso entre algunas personas con antepasados portugueses asentados desde finales del xix en algunas de estas poblaciones, por ejemplo, en Puerto La Laja (Sanlúcar de Guadiana) o El Romerano (San Silvestre de Guzmán). Tal vez la bibliografía más actualizada se encuentre en la tesis de López de Aberasturi (2015) y en los resultados del trabajo de campo que se ha venido realizando dentro del proyecto Frontespo y en el Simposio de Alcanena, en julio de 2016. Así como, viceversa, se han localizado españolismos en tierras algarvias en Vila Real de Santo Antonio, Castro Marim, Alcoutim, 
Laranjeiras, Monte Gordo, Junqueira, Azinhal Almada de Ouro. Contacto ${ }^{22}$ existe entre el leonés y el andaluz (López de Aberasturi 1986), entre el portugués y el andaluz (Alvar 1963) o entre el portugués y el español de Canarias ${ }^{23}$. Pueden consultarse trabajos recientes, sobre los contactos entre el portugués y el español a ambos lados del Atlántico, por ejemplo, en Amaral y Carvalho (2014), y también en África y Asia en Carrera de la Red (2012).

\section{Bibliografías no lingüísticas sobre la frontera hispano-portuguesa}

Recordemos que no son menos importantes los intercambios fronterizos de tipo etnográfico, antropológico o sociológico. Así, en lo que se refiere al área de contacto de Andalucía con el Alentejo y el Algarve no deben dejar de consultarse los trabajos que regularmente han publicado, ya se dijo, Elodia Hernández León, Ángeles Castaño Madroñal, Victoria Quintero y Rafael Cáceres, bajo proyectos como, entre otros, Cambios económicos, transformaciones socioculturales, etnicidad y simbolismo en Andalucía de la Universidad de Sevilla (1987-1992).

La frontera es también un polo de atracción turístico como se puede ver en el conjunto de interesantes trabajos con abundante bibliografía, incluidos en Campesino Fernández (Dir. 2013) o en Campesino Fernández y Jurado Almonte (Dirs. 2016), bajo el amparo de RIET (Rede Ibérica de Entidades Transfronteiriças) con sede en Vigo, y en el Proyecto TURFront (2000-2020), Turismo de frontera, del Ministerio de Economía y Competitividad. Fondo Europeo de Desarrollo Regional. Se proponen, asimismo, iniciativas culturales entre las poblaciones próximas como I Mostra internacional de cinema FRONTEIRAS, entre Ayamonte y Vila Real de Santo António; representaciones teatrales con escenificaciones de enfrentamientos entre españoles y portugueses, por ejemplo, Semblanza de dos voluntades de Isidro Leiva, en Valencia de Mombuey (Badajoz) en agosto de 2016; recreaciones de refriegas entre los mismos personajes, como la conocida Batalla de Salgadela, en Figueira de Castelo Rodrigo (Guarda), fronteriza con la provincia de Salamanca; Encuentro internacional de tamborileros, en Zamora; intercambios de literatura oral y tradicional; momentos de ocio en los viajes fluviales por los ríos de frontera, Miño, Duero, Tajo o Guadiana; en el uso de la tirolina entre Alcoutim y Sanlúcar del Guadiana en Huelva; la asistencia a las famosas corridas de toros de Barrancos; e incluso la existencia de Peñas taurinas como la hispano-lusa de Fuentes de Oñoro y Vilar Formoso (Simões 2008; Tuleski 2001; Navas 2011). La gastronomía reúne a los pueblos fronterizos en encuentros o publicaciones: la Feira dos enchidos e dos presuntos, en Barrancos; Saberes e sabores da Raia, en Mourão; Jornadas transfronterizas del gurumelo, en Villanueva del Fresno; Feria transfronteriza del aceite y el olivar, en Vilvestre (Salamanca) (Oliver 2005). El contrabando se ha convertido también en una actividad de ocio con abundante bibliografía (Medina 2004). Asistimos a la puesta en marcha de la Ruta del contrabando, entre Barrancos y Oliva de la Frontera (Badajoz); la Rota do Contrabando, entre Marvão (Portalegre) y La Fontañera (Cáceres); la Ruta contrabandista, entre Barrancos (Beja) y Encinasola (Huelva); la Ruta a caballo desde Penha Garcia en Idanha-a-Nova hasta la frontera (Rovisco 2011); Alcoutim e Sanlúcar de

22 Algunos de los casos de contacto entre el portugués y el español europeos se pueden encarar con la situación del portugués de Brasil y el español de Uruguay (véase bibliografía sobre el asunto en Carvalho 2014). Un estudio panorámico sobre las fronteras lingüísticas en el occidente de la Península puede encontrarse en Andrés-Díaz (2007).

Por ejemplo, véanse los trabajos de Morera Pérez (1994), Corbella Díaz y Medina López (1996) o Corbella (2016). 
Guadiana, entre castelos e estrelas. Caminhada nocturna (organizada por la Fundación Descubre). Por último, la situación comprometida de los lobos en zonas próximas portuguesas y españolas puede dar lugar, como otros muchos temas comunes, a encuentros entre poblaciones afectadas, como las Jornadas transfronterizas. Ecoturismo y desarrollo sostenible que, bajo el título de El lobo en España y Portugal, tuvieron lugar en Puebla de Sanabria en 2007.

El interés por la música en la raya se manifiesta, entre otros, por el proyecto de investigación de la Fundação para a Ciência e a Tecnologia, que lleva a cabo Dulce Simões desde 2013, A cultura expressiva na fronteira luso-espanhola: continuidade histórica e processos de transformação socioculturais, agentes e repertórios na construção de identidades (disponible en http://culturaexpressiva.wordpress.com) (Simões 2014; Godinho 2011). Existen testimonios como los Monumentos en el Guadiana al contrabandista en Sanlúcar del Guadiana (Huelva), frente al "guardinha", Guarda Fiscal, en Alcoutim (Faro). Asimismo, en Oliva de la Frontera (Badajoz) hay dos estatuas dedicadas a los mochileros. Pero también se encuentran memoriales a personajes históricos como al político portugués Humberto Delgado en Villanueva del Fresno (Badajoz), donde fue asesinado. La guerra civil española es un campo bibliográfico en el que abundan las relaciones humanas e históricas que se establecieron durante el conflicto. Nos referimos, entre otros, a los reunidos por Rosas (Coord.) (1998) con motivo del Coloquio que se celebró, en 1996, en el Instituto de Historia Contemporánea de la Faculdade de Letras da Universidade Nova de Lisboa, en el sexagésimo aniversario del estallido de la lucha fratricida. O los testimonios recogidos por Simões (2007) en la zona de Barrancos (Beja) sobre los españoles que huían de la represión franquista y que fueron acogidos en un campo de refugiados, todo ello dentro del proyecto general de la Asociación para la Recuperación de la Memoria Histórica.

El teatro puede ser también un punto de reflexión sobre estas lenguas amenazadas. Así el grupo teatral A Menor Língua do Mundo, dirigido por Alex Cassal y Paula Diogo, busca un espacio de conocimiento de las lenguas minoritarias como el minderico, el mirandés y el barranqueño con espectáculos que buscan en los lugares de origen de estas lenguas un espacio "privilegiado de criação e de debate sobre [...] a diversidade cultural, memória, democracia e liberdade".

Por último, no es extraño que se reflexione sobre otros aspectos más intangibles como la conciencia histórica, las ideas y mentalidades que circulan en este espacio de frontera; y sobre otros tan significativos como la propia identidad de cada comunidad frente a la otra. Sobre el primer significado, la conciencia histórica del área de la frontera, se recuperaron algunas reflexiones en el número de 2017 de la Revista de Histórias e Ideias, de la Universidad de Coimbra. Sobre el segundo, la propia identidad frente a la del vecino, se pueden consultar los trabajos, por ejemplo, de Amante (2007), Cairo et al. (coords.) (2009), Fernández (2019), Oliveira (2002), Pereiro, Risco y Llana (Orgs.) (2008) o Valcuende del Río (1998). Para completar este análisis conviene conocer las distintas denominaciones, motes, hipocorísticos, gentilicios, que los vecinos de los lugares próximos se atribuyen unos a otros. Es interesante, por último, en nuestra opinión, incluir asimismo en estos estudios bibliográficos las actitudes que sobre su propia lengua, sobre su propia cultura y sobre la de los vecinos manifiestan unas y otras poblaciones de la frontera. A modo de ejemplo se pueden consultar los trabajos de Iglesias Ovejero (1989) para la zona de Salamanca y Cáceres con Guarda. 


\section{A modo de conclusión}

Hemos presentado un recorrido a lo largo del tiempo de los distintos abordajes que se han acometido acerca de la frontera hispano-portuguesa, pero también hemos dado cuenta de las iniciativas más recientes. En lo que se refiere a estas últimas, a modo de reflexión final, convendría destacar los de gran impacto; por un lado, los Programas Europeos tales como los INTERREg, las ayudas de la Comisión Europea para el Fondo Europeo de Desarrollo Regional, los FEDER, el Programa Operativo de Cooperación Transfronteriza Portugal-España, las NUTs, la creación de las eurorregiones, Alentejo-Centro y Extremadura, y sobre todos los dos iniciativas más innovadoras y con más futuro que son las cinco Eurociudades proyectadas. En lo referente al ámbito de investigación universitaria, destacaríamos los dos $\mathrm{I}+\mathrm{D}+\mathrm{i}$ ajustados, uno al espectro geopolítico y antropológico de la frontera hispano-portuguesa, dirigido por Heriberto Cairo Carou de la Universidad Complutense de Madrid, el referente turismo de frontera, TURFRonT, responsables Campesino Fernández y Jurado Almonte, así como el más reciente, que está en curso, FRONTESPO-3P, relativo a aspectos culturales, lingüísticos y etnográficos, centrado en la recogida de materiales y bibliografía, y disponible siempre online, dirigido por Xosé Afonso Álvarez de la Universidad de Alcalá. También queremos destacar los trabajos referidos a las lenguas minoritarias, inmersos todos ellos en el proyecto que lleva a cabo el Centro Interdisciplinar de Documentação Linguística e Social (CIDLes), dirigido por Vera Ferreira, con sede en Alcanena (Santarém). Así mismo, queremos mencionar la intensa labor de contacto, aproximación, difusión e investigación que desarrollan las Universidades de Extremadura, Salamanca y de Huelva en la amplia variedad de cursos y publicaciones que llevan a cabo bajo sus distintos programas e iniciativas. Por último, entendemos que es imprescindible recordar la tarea difusora que ejerce virtualmente Rayanos Magazine en youtube así como los acercamientos constantes que las poblaciones de ambos lados de la frontera ponen en marcha, con el fin de unirse ante el hecho de su evidente aislamiento físico respecto de los poderes centrales, ya sea creando museos, realizando festivales, encuentros folklóricos o, entre otros, apoyos puntuales ante catástrofes como las derivadas de incendios o inundaciones.

\section{Referencias bibliográficas}

AA.VV. (2001). Revista de Filología Románica, 18. Número monográfico dedicado a las fronteras lingüísticas peninsulares.

Alonso de la Torre, J. R. (2006). La frontera que nunca existió. Viaje por la Raya de Extremadura y el Alentejo. Mérida: Editora Regional de Extremadura.

Alvar, M. (1963). Portuguesismos en andaluz. In Wltoffene Romanistik. Festschrift Alwin Kuhn zum 60. Geburstag. Innsbruck (pp. 309-324). <http://www.cervantesvirtual.com/obra-visor/portuguesismos-en-andaluz-0/html/010bd27e-82b2-11df-acc7-002185ce6064_7.html>

Amador Moreno, S. (2015). Español y portugués en contacto. Las actitudes lingüisticas en zona fronteriza: Extremadura y Alentejo. Cáceres: Universidad de Extremadura. Tesis doctoral, < file://C:/Users/Usuario/ Downloads/TDUEX_2016_Amador_Moreno.pdf>. 
Amante, M. F. (2007). Fronteira e identidad. Construção e representação identitárias na raia luso-espanhola. Lisboa: Instituto Superior de Ciências Sociais e Políticas.

Amaral, P.; \& Carvalho, A. M. (Eds.) (2014). Portuguese-Spanish Interfaces. Amsterdam-Philadelphia: John Benjamins.

Andrés-Díaz, R. de (2007). Linguistic borders of the Western Peninsula. International journal of the sociology of language, 184, 121-138.

Aunión, J. A. (2019). El rescate del tesoro de la raya. El País (30/5/2019).

Bastos, Ch. L. D.; Duarte Alves de Lara Everard, P. (1982). Da antropologia ao antropólogo: Barrancos de um Percurso. Lisboa: Universidade Nova de Lisboa. Tesis de licenciatura, inédita.

Bibliografia linguística portuguesa. Lisboa: Universidade Nova de Lisboa. Disponible en http://clunl.fcsh.unl. pt/bilp/ [Consulta: 07/09/2016].

Cáceres Feria, R.; \& Corbacho Gandullo, M. Á. (2013). Una propuesta para el análisis de la articulación social del litoral andaluz a través de la pesca. Revista andaluza de antropología, 4, 55-78.

Cáceres Feria, R.; Castaño Madroñal, Á; Hernández León, E.; \& Quintero Morón, V. (1993). Tres localidades fronterizas. Percepción y significación de la frontera. En Sistemas de identidades y su expresión en las sociedades locales, VI Congreso de Antropología, Federación de Asociaciones de Antropología del Estado Español (FAAEE) y Asociación Canaria de Antropología (pp. 53-64). Santa Cruz de Tenerife.

Cairo Carou, H.; \& Godinho, P. (2013). El Tratado de Lisboa de 1864: la demarcación de la frontera y las identificaciones nacionales. Historia y Política, 30, 23-54.

Cairo Carou, H.; Godinho, P.; \& Pereiro, X. (Coords.) (2009). Portugal e Espanha. Entre discursos de centro e práticas de frontera. Lisboa: Colibri/Instituto de Estudos de Literatura Oral.

Calderón Vázquez, F. J. (2015). Repasando la frontera hispano-portuguesa: conflicto, interacción y cooperación transfronteriza. Estudios fronterizos, 16, 31, 65-89.

Camacho, M.; Escoval, L.; Pereira, O.; Maldonado, M. do C.; \& Fradinho, F. (1999). Além da Água. Beja: Associação do Município do Distrito de Beja.

Campesino Fernández, A. J. (Dir.). (2013). Turismo de Frontera I. Eixo Atlântico do Noroeste Peninsular: Edições RIET.

Campesino Fernández, A. J.; \& Jurado Almonte, J. M. (Dirs.). (2016). Turismo de frontera (III). Productos turísticos en la raya ibérica. Huelva: Diputación de Huelva.

Candeias, M. F. S. (1998). O Alentejo e a Guerra Civil de Espanha: Vigilância e Fiscalização das Povoações Fronteiriças. Lisboa: Faculdade de Letras. Tesis de Maestría en Historia Moderna, inédita.

Carrasco González, J. M.; \& Viudas Camarasa, A. (Eds.). (1996). Actas del Congreso Internacional Luso-Español de Lengua y Cultura en la Frontera. 3 vols. Cáceres: Universidad de Extremadura.

Carrera de la Red, M. (2012). Lingüística románica. Conceptos y métodos. Lingüística del contacto entre el español y el portugués. Saarbrücken: Editorial Académica Española.

Carvalho, A. M. (2014). Sociolinguistic continuities in language contact situations: The case of Portuguese in contact with Spanish along the Uruguayan-Brazilian border. In P. Amaral, \& A. M. Carvalho (Eds.), Portuguese-Spanish Interfaces (pp. 263-294). Amsterdam-Philadelphia: John Benjamins.

Cayetano Rosado, M. (2018). La raya ibérica. De campo de batalla al de la emigración y otras cuestiones peninsulares. [Badajoz]: Fundación CB.

Clements, J. Clancy; \& Lorenzino, G. (2006). The contact situation in Barrancos, Portugal. Annual Meeting of the Society for Pidgin and Creole Languages. Alburquerque, NM, 5-8 de Janeiro. 
Corbella Díaz, D. (2016). Presencia del léxico gallego-portugués en el español atlántico: primeros testimonios. Estudos de lingüística galega, 8, 69-87.

Corbella Díaz, D.; \& Medina López, J. (1996). El contacto del portugués y el español en Canarias: estado de la cuestión. In J. M. Carrasco González, \& A. Viudas Camarasa (Eds.), Actas del Congreso Internacional Luso-Español de Lengua y Cultura en la Frontera (vol. I) (pp. 509-518). Cáceres: Universidad de Extremadura.

Cordero Torres, J. M. (1960). Fronteras hispánicas: geografía e historia, diplomacia y administración. Madrid: Instituto de Estudios Políticos.

Correia, V. (s.d.). Proposta de uma convenção ortográfica de uma língua de contacto no séc. xxı: Aplicação ao barranquenho. Évora: Universidade de Évora. Tesis doctoral, en elaboración.

Domingues, C. M. (1995). Fronteira, como ponto de união. Beja: Universidade Moderna de Beja. Seminario de Antropología, inédito.

- (2003). Barrancos e Encinasola: Fronteira e Destino. Évora: Universidade de Évora. Tesis de Maestría en Sociología, inédita.

Fernández, M. J. (2019). Iberia cara a cara: Mirar sin verse. Las relaciones luso-españolas desde la imagología literaria. En L. F. de la Macorra y Cano (Coord.), Convergencia Ibérica. ¿"Quo Vadis" Iberia? (pp. 17-49). Badajoz: Diputación de Badajoz. <https://www.academia.edu/41327538/Mirar_sin_verse._Las_relaciones_luso-espa\%C3\%B1olas_desde_la_Imagolog\%C3\%ADa_Literaria>

Ferreira, M. B. (Coord.) (2006). Língua e história na fronteira Norte-Sul. Bibliografia. <http://www.geolectos. com/livro.pdf $>$.

. (Coord.) (2010). Língua e cultura na fronteira Norte-Sul. Bibliografia. <http://www.geolectos. com/livro.pdf $>$.

Ferreira, V.; Schulze, I.; Carvalho, P. V.; \& Bouda, P. (2015). Dicionário bilingue. Piação-Português. Fátima: Centro Interdisciplinar de Documentação Linguística e Social (cidLes).

García Álvarez, J. (Ed.) (2015). La frontera hispano-portuguesa. Representaciones geográficas, paisajes y delimitación territorial (siglos XVII-XXI). Revista de historiografía, 23, 2.

Gargallo Gil, J. E. (1999). Las hablas de San Martín de Trevejo, Eljas y Valverde del Fresno. Trilogía de los tres lugares. Mérida: Editora Regional de Extremadura.

Godinho, P. (2011). Identificacións locais, culturas das marxes e construción de nacións na fronteira entre Portugal e Galicia. Ourense: Diputación de Ourense. <http://hdl.handle.net/10362/14518>.

- (2015). «Fronteiras e cicatrices da história num tempo de comemorações». En I Congreso Internacional sobre Fronteiras Comparadas, Pontevedra, 24 y 25 de septiembre de 2015. <http://tv.uvigo.es/ matterhorn/27825>.

Gualda Caballero, E. (Dir.) (2008). Realidad social en Andalucía, Algarve y Alentejo. Huelva: Publicaciones de la Universidad de Huelva. <https://estrellagualda.es/descargas/publicaciones/2008_Realidad_Social_ AAA.pdf $>$.

Hernández León, E.; \& Castaño Madroñal, Á. (1992-1993). Expresiones simbólicas y cultura de fronteras en la raya de Portugal. Anuario etnológico de Andalucía, 3, 217-222.

Heredero de Pablos, M. I.; \& Olmedillas Blanco, B. (2009). Las fronteras españolas en Europa: de INTERREG a la cooperación territorial europea. Investigaciones regionales, 16, 191-125.

Iglesias Ovejero, Á. (1989). El blasón popular de los Farinatos y sus aledaños: Ciudad Rodrigo, El Rebollar y Campo de Azaba y Argañán. In P. Peira, P. Jauralde, J. Sánchez Lobato, \& J. Urrutia (Orgs.), Homenaje a Alonso Zamora Vicente. Dialectología. Estudios sobre el Romancero (vol. 2) (pp. 171-187). Madrid: Castalia. 
Jacinto, R.; \& Cabero Diéguez, V. (Coords.) (2014). Espaços de fronteira, territórios de esperança. Paisagens e património, permanências e mobilidades. Guarda: Centro de Estudos Ibéricos. Lisboa: Âncora Editora.

Lopes, J. E. (2001). Barrancos: Identidade Cultural. Lisboa: Instituto Superior de Ciências do Trabalho e da Empresa. Tesis de Licenciatura en Sociología, inédita.

López Martínez, A. L. (2004). La presencia portuguesa en el litoral occidental onubense. Huelva en su historia, 11, 187-202.

López de Aberasturi Arregui, I. (1986). Introducción al estudio de leonesismos y portuguesismos en andaluz. Granada: Universidad de Granada. Tesina inédita.

- (2015). Dinámica sociolingüística y lenguas en contacto en la comunidad de Ayamonte. Granada: Universidad de Granada. Tesis doctoral. <https://hera.ugr.es/tesisugr/26507936.pdf>

Lorenzana de la Puente, F. \& Mateos Arcacíbar, F. J. (Coords.) (2008). Iberismo. Las relaciones entre España y Portugal. Historia y tiempo actual y otros estudios sobre Extremadura. Llerena: Sociedad Extremeña de Historia.

Marcos de Dios, Á. (Ed.) (2007). Aula ibérica. Salamanca: Universidad de Salamanca.

Martín Galindo, J. L. (1999). A fala de Xálima. O falar fronteirizo de Eljas, San Martín de Trevejo y Valverde. Mérida: Editora Regional de Extremadura.

Martínez Reñones, J. A. (2011). La Raya. Una frontera absurda. Un viaje a la frontera hispano-portuguesa. León: Lobo Sapiens.

Medina García, E. (2004). Contrabando en la frontera de Portugal: Orígenes, estructuras, conflicto y cambio social. Madrid: Servicio de Publicaciones de la Universidad Complutense de Madrid.

Morera Pérez, M. (1994). Español y portugués en Canarias. Puerto del Rosario: Servicio de Publicaciones del Cabildo Insular de Fuerteventura.

Nagore Laín, F.; \& Puig López, P. (Eds.) (1999). Luengas menazatas y normalización. Huesca: Consello d’a Fabla Aragonesa.

Navas Sánchez-Élez, M. V. (1998). La frontera lingüística hispano-portuguesa: aproximación bibliográfica. Madrygal, 1, 83-89.

— (1999). La frontera lingüística hispano-portuguesa: aproximación bibliográfica (Cont.). Madrygal, 2, 115-116.

. (2011). El barranqueño: un modelo de lenguas en contacto. Madrid/Lisboa: UCM/CLuL.

. (2012). Isabel Faria sí estuvo en Barrancos. In A. Costa, \& I. Duarte (Coords.), Nada na linguagem lhe é estranho. Homenagem. Isabel Faria (pp. 619-634). Lisboa: Edições Afrontamento.

- (2014). Presente, pasado y futuro de una lengua de contacto lusoespañola. En J. M. Santos Rovira (Ed.), Fronteras y diálogos el español y otras lenguas (pp. 89-100). Lisboa: Axac.

. (2015). El barranqueño, lengua oral versus lengua estándar: estado de la cuestión. Luenga y fablas, $19,83-89$.

. (2017). Intercambios culturales y lingüísticos en la raya hispanoportuguesa: estado de la cuestión. In D. Corbella, \& A. Fajardo (Eds.), Español y portugués en contacto: préstamos léxicos e interferencias, Número monográfico de Zeitschrift für romanische Philologie (BZrP) (pp. 79-104). Berlín/Boston: de Gruyter.

Oliver Servera, T. (2005). Entre pucheros, marochos y barranqueños: histórica gastronómica de Encinasola y Barrancos. Ayuntamiento de Encinasola, Ayuntamiento de Barrancos: Junta de Andalucía.

Oliveira, S. M. de (2002). Discourses of Identity at the Spanish/Portuguese Border: Self-Identification Strategies of Centre and Periphery. National Identities 4, 3, 245-256. 
Ossenkop, C. (2010). Contacto de lenguas en la frontera luso-extremeña: la situación del español y del portugués en la franja fronteriza de Cedillo, Valencia de Alcántara y La Codosera. In M. J. Fernández García, \& S. Amador Moreno (Eds.), Al límite (pp. 5-14). Cáceres: Avuelapluma. <http://seeplu.galeon.com/textos1/ christina.html>.

Pereiro, X.; Risco, L.; \& Llana, C. (Orgs.) (2008). As fronteiras e as identidades raianas entre Portugal e España. Vila Real: UTAD.

Pintado García, A.; \& Barrenechea. E. (1972). La raya de Portugal. La frontera del subdesarrollo. Madrid: Cuadernos para el Diálogo.

Pires, J. C. S. U. (1997). A Memória da Guerra Civil de Espanha no Baixo Alentejo Raiano. Lisboa: Instituto Superior de Ciências do Trabalho e da Empresa. Máster en Historia Social Contemporánea, inédito.

Quijada Coronel, B. (2012). Patrimonio cultural en la frontera Alentejo-Extremadura. Las nanas, ¿una tradición oral viva? Évora: Universidade de Évora. Máster, inédito.

Revez, J.; \& Cascalheira, S. (Coords.) (2010). Do saber ao contar. Mértola: Associação do Património de Mértola. . (Coords.) (2010). Del saber al contar. Mértola: Associação do Património de Mértola.

Rey Yelmo, J.C. (1999). A fala. La fala de San Martín de Trevejo. O mañegu. Mérida: Editora Regional de Extremadura.

Rodrigues, J. T. H. (2013). O Contrabando no Baixo Guadiana - A Raia, as Gentes e as Dimensões da Sobrevivência. Gambelas: Universidade do Algarve. Máster, inédito.

Rosas, F. (Coord.) (1998). Portugal e a guerra civil de Espanha. Lisboa: Colibri.

Rovisco, E. (2011). Fronteira e turismo no "concelho mais português de Portugal". Geopolítica(s), 12, 1, 91-107.

Serradilla Castaño, A. (2006). Morfología y sintaxis extremeñas en el norte de Huelva. Fronteras lingüísticas vs fronteras políticas. Cahiers du P.R.O.H.E.M.I.O., 7, 131-148.

Silva, L. M. S. (1999). Identidade nacional: Práticas e representações num contexto de frontera. Lisboa: ISTCE. Tesis de Maestría Antropología, Património e Identidades, inédita.

Simões, M. D. A. (2007). Barrancos na encruzilhada da Guerra Civil de Espanha. Memórias e testemunhos 1936. Câmara Municipal de Barrancos: Edições Colibri.

. (2008). Fronteras estatales y relaciones sociales en la frontera hispano-portuguesa. El caso de Barrancos y Oliva de la Frontera». Gaceta de Antropología, 24, 2. <http://www.ugr.es/ pwlac/G24_52MariaDulce_Antunes_Simoes.html>.

. (2014). Práticas musicais e performativas na fronteira luso-espanhola: conexões transnacionais e re-significações locais. In L. Martin, U. Martínez Veiga, \& J. Palomera (Coords.), Periferias, Fronteras y Diálogos. Simposio: Transnacionalismo, interconexiones geográficas y fronteras en tiempos de crisis (pp. 4151-4167). Tarragona: Universitat Rovira i Virgili.

Stefanova-Gueorguiev, I. (2000). Español y portugués en la Península Ibérica y en América Latina: dos situaciones de contacto lingüístico. Simon Fraser University (Latin American Studies Program, Department of Sociology and Anthropology). Tesis doctoral, inédita. <http://www.collectionscanada.gc.ca/obj/s4/f2/ dsk2/ftp01/MQ61500.pdf>.

Tuleski, V. E. R. (2001). Touros de norte em Barrancos: uma Tradição da Fronteira Luso-Espanhola. Lisboa: Insituto Superior de Ciências do Trabalho e da Empresa. Máster, inédita.

Valcuende del Río, J. M. (1998). Fronteras, territorios e identificaciones colectivas, interacción social, discursos políticos y procesos identitarios en la frontera sur hispano-portuguesa. Sevilla: Fundación Blas Infante. 